\title{
Contribución al estudio de los triatominos del Perú: Distribución geográfica, nomenclatura y notas taxonómicas
}

\author{
Julio Chávez ${ }^{1}$
}

Resumen

Palabras clave
Objetivos: Determinar el número de especies válidas, distribución geográfica, sinonimias, nomenclatura y comentarios taxonómicos de la subfamilia Triatominae. Materiales y Métodos: Se compiló la lista de nombres científicos y distribución geográfica de triatominos existentes en el Perú en las referencias bibliográficas presentadas. Resultados: Existen 18 especies de la subfamilia Triatominae en el Perú distribuidos en 23 departamentos; todas ellas agrupadas en 4 principales tribus y 8 géneros. Conclusiones: Es necesario realizar estudios sobre ecología, morfología, biología molecular y epidemiología para una identificación precisa de especies de triatominos que existen en Perú.

Triatominae; apuntes tripanocidas; tripanosomiasis; enfermedad de Chagas.
Contribution to the study of Triatominae in Peru: Geographic distribution, nomenclature and taxonomic notes

Abstract

Objectives: To determine the number of valid species, geographical distribution, synonymies, nomenclature and taxonomic commentaries of the Triatominae subfamily. Materials and Methods: Using the references presented below we collected the list of scientific names and geographical distribution of Triatominae existing in Peru. Results: There are 18 species of Triatominae sub-family in Peru distributed in 23 departments and grouped in 4 main tribes and 8 genders. Conclusions: It is necessary to performecology, morphology, molecular biology and epidemiology studies for a precise identification of the Triatominae species existing in Peru.

Keywords: Triatominae; trypanocidal agents; tryoanosomiasis; Chagas disease.

\section{INTRODUCCIÓN}

Los triatominos son vectores que transmiten Trypanosoma cruzi Chagas, 1909, el agente causante de la enfermedad de Chagas o tripanosomiasis americana $\left({ }^{1}\right)$. Se

\footnotetext{
Instituto de Investigación en Microbiología y Parasitología Tropical, Facultad de Ciencias Biológicas, Universidad Nacional de Trujillo. Trujillo, Perú.
}

estima que alrededor de 650000 a 680000 personas, pueden ser infectadas por $T$. cruzi en el Perú, con 5 a 6,8 millones de personas que viven en riesgo ${ }^{2}$ ).

En el Perú, se conoce un total de 7 géneros con 18 especies de triatominos $\left({ }^{3}\right)$, a los cuales se divide en 3 grupos, según su hábitat: a) domésticos, que viven en la vivienda; b) peridomésticos, que viven en cuyeros o gallineros; y, c) extradomésticos o silvestres, que viven en los nidos y cuevas de otros animales $\left({ }^{4}\right)$.

La clasificación de la subfamilia Triatominae Jeannel, 1919, está basada principalmente en estudios de características morfológicas realizadas a las diferentes especies y presentadas en el Boletín del Museo Americano de Historia Natural $\left({ }^{5}\right)$.

Hoy en día, la genética y biología molecular son fundamentales en el área de la sistemática, ya que nos permite contestar ciertas interrogantes al momento de clasificar y determinar las especies, tanto a nivel interespecífico como intraespecífico. Actualmente, el concepto de especie está continuamente siendo redefinido y 
comúnmente muchos investigadores prefieren el concepto de población que el de especie $\left(^{6}\right)$.

El propósito del presente estudio es clarificar el estado actual de los nombres de especies válidas en la subfamilia Triatominae del Perú.

\section{MATERIALES Y MÉTODOS}

La lista de nombres científicos de las especies de triatominos fue compilado de la literatura que se presenta en las referencias bibliográficas. El criterio usado para considerar a especies como válidas se basa solo en especies reconocidas por sus características morfológicas y/o moleculares demostradas. No se considera propuestas de cambios taxonómicos basadas solo en datos moleculares, pero se presenta comentarios.

Se elaboró un mapa del Perú señalando las especies de triatominos reportadas (Figura 1).

\section{RESULTADOS}

Lista de especies presentes para el Perú: Sinonimia, distribución geográfica y hábitat.

\section{Reino Animalia \\ Filo Arthropoda \\ Clase Insecta \\ Orden Hemíptera}

Familia Reduviidae

Subfamilia Triatominae Jeannel, 1919( $\left.{ }^{7}\right)$

Tribu Bolboderini Usinger, 1944( $\left.{ }^{8}\right)$

Género Belminus Stål, $1859\left(^{9}\right)$

Sinónimo: Marlianus Distant, $1902\left({ }^{10}\right)$.

Belminus peruvianus Herrer, Lent \& Wygodzinsky, 1954( $\left.{ }^{7}\right)$.

Distribución geográfica en el mundo $\left({ }^{6}\right)$ : especie endémica del Perú.
Distribución geográfica en el Perú $(2,6,11,12,13,14)$ : Cajamarca (Jaén), Amazonas (Bagua, Utcubamba, Lonya Grande). Hábitat: silvestre

Género Microtriatoma Prosen \& Martínez, 1952 (15).

Microtriatoma trinidadensis (Lent, 1951) $\left({ }^{16}\right)$ Prosen \& Martínez, $1952\left({ }^{17}\right)$.

Sinónimos: Bolbodera trinidadensis Lent, $1951{ }^{\left({ }^{16}\right)}$; Microtriatoma mansosotoi Prosen \& Martínez, $1952\left({ }^{15}\right)$.

Distribución geográfica en el mundo $\left.{ }^{6}\right)$ : Brasil (Pará), Bolivia (Beni, Cochabamba, La Paz, Santa Cruz), Colombia (Meta), Trinidad, Venezuela (Sucre, Dealta, Amacuro).

Distribución geográfica en el Perú $\left({ }^{2,6,14}\right)$ : Cusco (Quispicanchi, Valle del Monsón).

Hábitat: Silvestre.

Tribu Cavernicolini Usinger, $1944\left(^{(8)}\right.$

Género Cavernicola Barber, 1937( $\left.{ }^{18}\right)$

Cavernicola pilosa Barber, 1937( $\left.{ }^{18}\right)$

Sinónimos: Cavernicola pilosus Barber, $1937\left({ }^{18}\right)$.

Distribución geográfica en el mundo $\left.{ }^{(}\right)$: Brasil (Bahía, Espírito Santo, Mato Grosso, Pará, Paraná), Colombia (Cundinamarca, Meta, Tolima, Valle), Panamá, Venezuela (Cojedes, Portuguesa, Táchira, Lara, Amazonas). Distribución geográfica en el Perú $\left({ }^{2,6,12}\right)$ : Loreto (Iquitos, Francisco de Orellana), Cajamarca (Celendín), La Libertad (Pataz).

Hábitat: silvestre, presente en árboles con nidos de murciélagos.

Tribu Rhodniini Pinto, $1926\left({ }^{19}\right)$

Género Rhodnius Stål, $1859\left({ }^{9}\right)$

Rhodnius ecuadoriensis Lent \& León $1958\left({ }^{20}\right)$ 


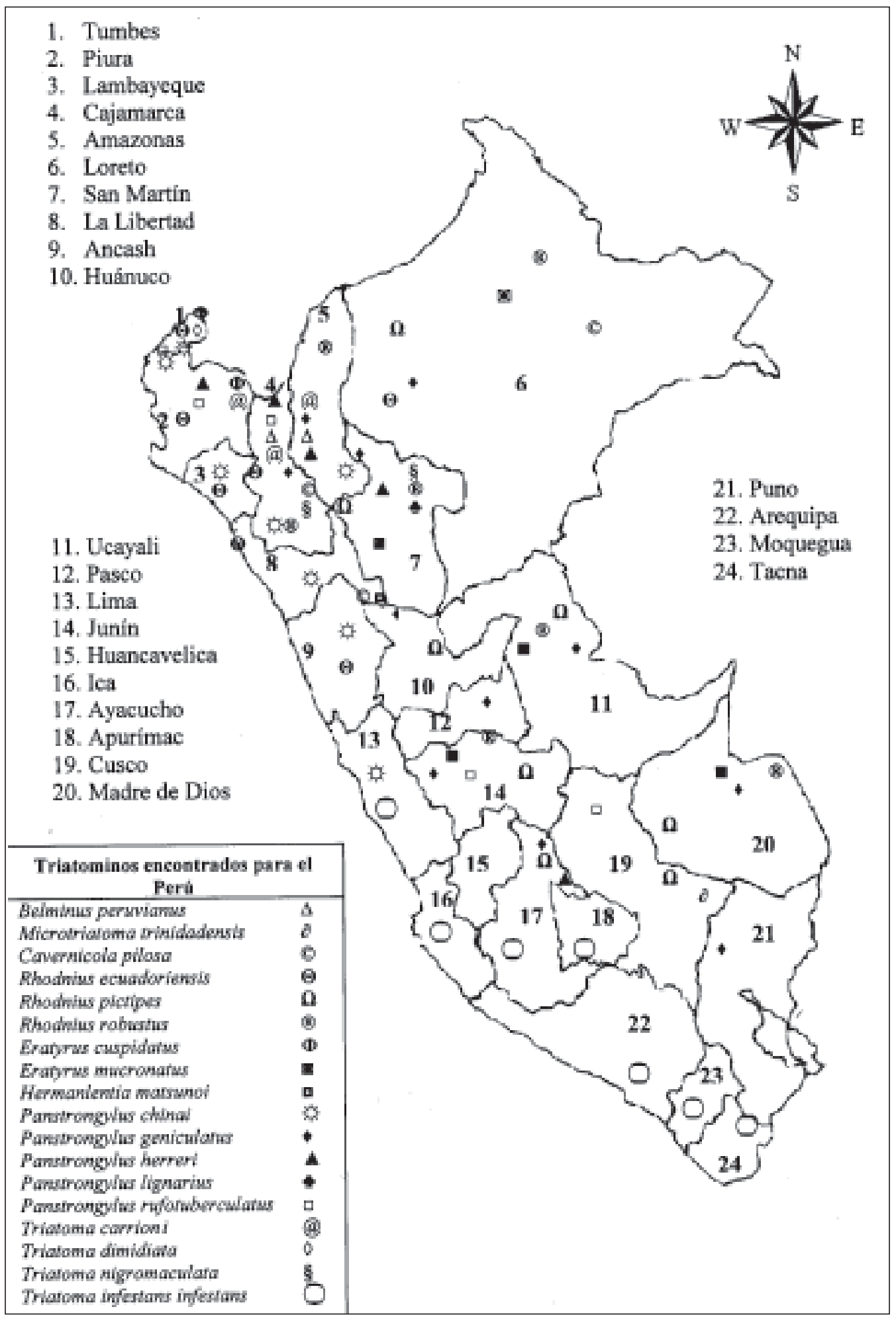

Figura 1. Presencia de triatominos en los departamentos del Perú. 
Distribución geográfica en el mundo $\left({ }^{6}\right)$ : Sur de Colombia, Ecuador (Manabí, Guayas, Loja).

Distribución geográfica en el Perú (2,6,11,12,14,21,22): Tumbes (Tumbes, Zarumilla), Piura (Ayabaca, Suyo, Huancabamba, Morropón, Piura), Lambayeque (Ferreñafe, Lambayeque, Chiclayo), Cajamarca (Jaén, Cutervo, Chota, San Miguel, Celendín, Cajamarca, Contumazá, San Benito), La Libertad (Trujillo, Chicama, Compín, Otuzco, Cascas, Gran Chimú), Ancash (Aija).

Hábitat: doméstico y peridoméstico; en Piura, se le encontró en Schinus mollis 'molle'.

Rhodnius pictipes Stål, $1872\left({ }^{23}\right)$

Sinónimos: Conorhinus limosus Walker, $\left.1873{ }^{(24}\right)$.

Distribución geográfica en el mundo $\left({ }^{6}\right)$ : Belice, Brasil (Amazonas, Goiás, Maranhão, Pará), Colombia (Meta, Putumayo), Ecuador (Azuay), Guyana, Guyana Francesa, Surinam, Trinidad, Venezuela (Anzoátegui, Apure, Aragua, Bolívar, Carabobo, Cojedes, Falcón, Mérida, Miranda, Monagas, Portuguesa, Táchira, Trujillo, Sucre, Yaracuy, Zulia, Delta, Amacuro).

Distribución geográfica en el Perú $(2,6,11,13,14,25)$ : Loreto (Yurimaguas, Loreto, Maynas, Alto Amazonas, Requena), Ucayali (Coronel Portillo), San Martín (San Martín, Mariscal Cáceres, Huallaga, Rioja, Lamas), Cusco (Quispicanchi), Madre de Dios (Tahuamanú, Manú), Huánuco (Leoncio prado, Dos de Mayo), Junín (Satipo, Tarma), Ayacucho (Huanta, la Mar).

Hábitat: silvestre, en palmeras; algunas colonias domésticas en San Martín.

Rhodnius robustus Larrousse, $1927\left({ }^{26}\right)$ Distribución geográfica en el mundo $\left.{ }^{6}\right)$ : Bolivia (Pandos, Santa Cruz), Brasil (Amazonas, Goiás, Maranhão, Pará), Colombia (Santander, Norte Santander), Ecuador (Napo), Guyana Francesa, Venezuela (Mérida, Falcón, Táchira, Cojedes, Apure, Barinas, Bolívar, Monagas, Sucre, Trujillo, Yaracuy).

Distribución geográfica en el Perú $(2,6,11,12,13,14,25)$ : Loreto (Alto Amazonas, Loreto, Maynas, Alto y Bajo Ucayali), Ucayali (Coronel Portillo), San Martín (Lamas), Amazonas (Condorcanqui), Cajamarca (Cajamarca, Condebamba), Madre de Dios (Tahuamanú, Tambopata), Junín (Chanchamayo).

Hábitat: silvestre, en palmeras.

Tribu Triatomini Jeannel, $1919\left({ }^{27}\right)$

Género Eratyrus Stål, $1859\left({ }^{9}\right)$

Eratyrus cuspidatus Stål, $1859\left({ }^{9}\right)$

Distribución geográfica en el mundo $\left.{ }^{6}\right)$ : Colombia (Boyacá, Norte de Santander, Sucre), Ecuador (Esmeraldas, Loja), Guatemala, México (Chiapas), Panamá, Venezuela (Anzoátegui, Aragua, Falcón, Trujillo, Yaracuy, Táchira, Sucre, Zulia).

Distribución geográfica en el Perú $(2,6,12)$ : Tumbes (Zarumilla), Piura (Ayabaca).

Hábitat: silvestre.

Eratyrus mucronatus Stål, $1859\left({ }^{9}\right)$

Sinónimos: Conorhinus mucronatus Walker, $1873{ }^{(24)}$.

Distribución geográfica en el mundo $\left({ }^{6}\right)$ Bolivia (Beni, La Paz), Brasil (Amazonas, Goiás, Maranhão, Mato Grosso, Pará), Colombia (Meta, Norte Santander), Ecuador (Esmeraldas), Guatemala, Guyana, Guyana Francesa, Panamá, Surinam, Trinidad, Venezuela (Yaracuy, Aragua, Barinas, Carabobo, Guárico, Mérida, Monagas, Portuguesa, Zulia, Cojedes, Falcón, 
Lara, Sucre, Trujillo, Táchira).

Distribución geográfica en el Perú $(2,6,11,13,14,25)$ : Loreto (Maynas), Ucayali (Coronel Portillo), San Martín (Huallaga, Lamas, Mariscal Cáceres), Madre de Dios (Tambopata, Tahuamanu), Junín (Chanchamayo).

Hábitat: silvestre.

Género Hermanlentia Jurberg \& Galvão, $1997\left({ }^{28}\right)$

Hermanlentia matsunoi (Fernández Loayza, 1989) $\left({ }^{29}\right.$ ) Jurberg \& Galvão, $1997{ }^{(28)}$

Sinónimos: Triatoma matsunoi Fernández Loayza, $1989\left({ }^{29}\right)$.

Distribución geográfica en el mundo $\left.{ }^{6}\right)$ : especie endémica del Perú.

Distribución geográfica en el Perú $(2,6,12)$ : La Libertad (Pataz)

Hábitat: silvestre, en cuevas.

Género Panstrongylus Berg, $1879\left({ }^{30}\right)$ Sinónimos: Lamus Stål, $1859\left({ }^{9}\right)$; Mestor Kirkaldy, $1904{ }^{\left({ }^{31}\right)}$

Panstrongylus chinai (Del Ponte, 1929) ( $\left.{ }^{32}\right)$ Pinto, $1931\left(^{9}\right)$

Sinónimos: Triatoma chinai Del Ponte, 1929( $\left.{ }^{11}\right)$; Panstrongylus turpiali Valderrama, Lizano, Cabello \& Valera, $1996{ }^{\left({ }^{33}\right)}$.

Distribución geográfica en el mundo $\left({ }^{6}\right)$ : Ecuador (Loja), Venezuela (Zulia).

Distribución geográfica en el Perú $(2,6,11,12,13,14,21,25,34)$ : Tumbes (Tumbes, Zarumilla, Comandante Villar), Piura (Huancabamba, Ayabaca, Morropón, Paita, Piura, Sullana, Talara), Lambayeque (Lambayeque, Zaña, Reque, Salas, Ferreñafe, Chiclayo), La Libertad (Trujillo, Moche, Virú, Chao, Jequetepeque, Huamachuco, Otuzco, Bolívar, Chicama, Chepén, Chamán, Simbal, Pacasmayo, Pataz, Santiago de Chuco), Cajamarca (Cajamarca, Contumazá, Celendín, Santa Cruz,
Jaén, San Ignacio, Cutervo, Cajabamba, San Miguel), Amazonas (Bagua, Rodríguez de Mendoza, Chachapoyas, Utcubamba, Luya), Lima (Chancay, Huaral), Ancash (Santa, Casma, Huarmey, Huaylas, Yungay, Aija, Bolognesi, Recuay, Huaraz, Sihuas).

Hábitat: peridoméstico, en algunas zonas domésticos y silvestres.

Panstrongylus geniculatus (Latreille, 1811) ( $\left.{ }^{35}\right)$ Pinto, $1931\left({ }^{17}\right)$

Sinónimos: Conorhinus corticalis Walker, $1873 \quad\left({ }^{24}\right)$; Conorhinus geniculatus Walker, 1873( $\left.{ }^{63}\right)$; Lamus geniculatus Stål, $1859\left({ }^{9}\right) ;$ Mestor geniculatus Brindley, $1931\left(^{6}\right)$; Reduvius geniculatus Latreille, $1811\left({ }^{35}\right)$; Conorhinus lutulentus Erichson, 1848 ${ }^{(36)}$; Triatoma fluminensis Neiva \& Pinto, $\left.1922{ }^{(37}\right)$; Triatoma geniculata Chagas, 1912 ( $\left.{ }^{38}\right)$; Panstrongylus parageniculatus Ortiz, $1971{ }^{\left({ }^{39}\right)}$; Triatoma tenuis Neiva, $1914\left({ }^{40}\right)$.

Distribución geográfica en el mundo $\left.{ }^{6}\right)$ : Argentina (Corrientes, Chaco, Entre Ríos, Formosa, Misiones, Santa Fé, Santiago del Estero), Bolivia (Beni, Tarija, Santa Cruz), Brasil (Acre, Amapá, Amazonas, Bahía, Brasilia DF, Ceará, Espírito Santo, Goiás, Maranhão, Mato Grosso, Mato Grosso del Sur, Minas Gerais, Pará, Paraná, Rio de Janeiro, Rondônia, São Paulo), Colombia (Antioquia, Cauca, Cundinamarca, Huila, Norte Santander, Putumayo, Santander, Secre, Tolima, Valle), Costa Rica, Ecuador (Manabí, Imbabura, Pastaza), Guatemala, Guyana, Guyana Francesa, México (Yucatán, Chiapas, Veracruz), Nicaragua, Panamá, Paraguay (Concepción, Caaguazú, Paraguari, Alto Oparaná, Nueva Asunción), Surinam, Uruguay, Trinidad, Venezuela (Anzoátegui, Aragua, 
Bolívar, Carabobo, Falcón, Guárico, Lara, Mérida, Miranda, Monagas, Táchira, Yaracuy, Zulia, Distrito Federal, Delta Amacuro).

Distribución geográfica en el Perú $(2,6,11,12,13,14,21,25)$ : Loreto (Alto Amazonas, Maynas, Yurimaguas), Cajamarca (Jaén, San Ignacio, Cutervo), Junín (Chanchamayo, Satipo, Tarma), Amazonas (Bagua, Utcubamba), San Martín (Moyabamba, Mariscal Cáceres, Lamas), Ucayali (Coronel Portillo), Madre de Dios (Tahuamanú), Huánuco (Marañón, Leoncio Prado), Pasco (Pasco, Oxapampa), Ayacucho (Huanta, La Mar), Puno.

Hábitat: silvestre.

Panstrongylus herreri Wygodzinsky, $1948\left({ }^{41}\right)$

Distribución geográfica en el mundo $\left({ }^{6}\right)$ : Ecuador .

Distribución geográfica en el Perú (2,6,11,12,13,14,21,25): San Martín (Moyabamba, Rioja, Calzada, Yantaló, Picota, Lamas), Amazonas (Bagua, Rodríguez de Mendoza, Utcubamba, Lonya Grande, Luya), Cajamarca (Jaén, Cutervo, San Ignacio, Santa Cruz), Piura (Ayabaca), Ayacucho (La Mar). Hábitat: doméstico; en Piura, se encuentra colonias peridomésticas.

Panstrongylus lignarius (Walker, 1873) ${ }^{(24)}$ Pinto, $1931\left({ }^{17}\right)$

Sinónimos: Triatoma lignarius Walker, $1873\left({ }^{24}\right)$.

Distribución geográfica en el mundo $\left({ }^{6}\right)$ : Brasil (Amazonas, Goiás, Maranhão, Pará), Guyana, Surinam, Venezuela (Portuguesa).

Distribución geográfica en el Perú (2,6,13): San Martín (Lamas)

Hábitat: silvestre

Panstrongylus rufotuberculatus (Champion, 1899) ${ }^{42}$ )
Sinónimos: Triatoma coxo-rufa Campos, $1932 \quad\left({ }^{43}\right) ; \quad$ Triatoma rufotuberculata Neiva, $1914 \quad\left({ }^{44}\right)$; Lamus rufotuberculatus Champion, $1899\left({ }^{42}\right)$; Mestor rufotuberculatus Usinger, $1939\left({ }^{45}\right)$.

Distribución geográfica en el mundo ${ }^{(}{ }^{6}$ : Argentina (Jujuy), Bolivia (La Paz, Beni, Santa Cruz), Brasil (Amazonas, Mato Grosso, Pará), Colombia (Antioquia, Cauca, Meta, Valle), Costa Rica, Ecuador (Manabí, Guayas, El Oro, Imbabura, Pichincha, Loja), México (Chiapas), Panamá, Venezuela (Aragua, Carabobo, Cojedes, Falcón, Guárico, Lara, Monagas, Portuguesa, Yaracuy, Zulia, Sucre, Táchira, Distrito Federal, Amacuro).

Distribución geográfica en el Perú $(2,6,11,12,13,14)$ : Tumbes (Tumbes, Comandante Villar, Zarumilla), Piura (Piura, Ayabaca), Cajamarca (Jaén), Cusco (Convención), Junín (Chanchamayo, Tarma).

Hábitat: silvestre y peridoméstico.

Género Triatoma Laporte, $1832\left({ }^{46}\right)$

Sinónimos: Conorhinus Laporte, 1833 $\left({ }^{46}\right)$; Eutriatoma Pinto, $1926\left({ }^{39}\right)$; Neotriatoma Pinto, $1931\left({ }^{17}\right)$.

Triatoma carrioni Larrouse, $1926\left({ }^{47}\right)$ Sinónimos: Eutriatoma carrioni Pinto, $\left.1931{ }^{(17}\right)$

Distribución geográfica en el mundo $\left({ }^{6}\right)$ : Ecuador (El Oro, Cañar, Azuar, Zamora-Chinchipe).

Distribución geográfica en el Perú $(2,6,11,12,14)$ : Piura (Huancabamba, Ayabaca), Cajamarca (Jaén, Santa Cruz, San Miguel, Cutervo, Chota), Amazonas (Bagua).

Hábitat: doméstico y peridoméstico.

Triatoma dimidiata (Latreille, 1811) ${ }^{\left({ }^{35}\right)}$

Sinónimos: Triatoma capitata Usinger, $1941\left(^{48}\right)$; Triatoma dimidiata capitata 
Usinger, $1944\left({ }^{8}\right) ; \quad$ Conorhinus dimidiatus Stål, $1859\left({ }^{9}\right)$; Conorrhinus dimidiatus Champion, $1899 \quad\left({ }^{42}\right)$; Reduvius dimidiatus Latreille in Humboldt \& Bonpland, $1811{ }^{(35)}$; Conorhinus dimidiatus var. maculipennis Champion, $1899\left({ }^{42}\right)$; Conorhinus dimidiatus maculipennis Neiva, $1914 \quad\left({ }^{44}\right) ; \quad$ Conorhinus maculipennis Stål, $1859\left({ }^{9}\right)$; Triatoma maculipennis Pinto, $1931\left({ }^{17}\right)$.

Distribución geográfica en el mundo ${ }^{6}$ ): Belize, Colombia (Cundinamarca, Boyacá, Huila, Norte Santander, Magdalena, Casanare, Santander), Costa Rica, Ecuador (Guayas, Manabí, Esmeralda, El Oro, Los Ríos, Bolívar, Morona Santiago), El Salvador, Guatemala, Honduras, Mexico (Campeche, Chiapas, Colima, Guerrero, Jalisco, Nayarit, Oaxaca, Puebla, Quintana Roo, San Luis Potosí, Tabasco, Veracruz, Yucatan), Nicaragua, Venezuela (Carabobo, Cojedes, Yaracuy, Bolívar, Falcón, Delta Amacuro, Distrito Federal).

Distribución geográfica en el Perú $\left({ }^{2,6,12,14}\right)$ : Tumbes (Tumbes, Zarumilla), La Libertad (Pacasmayo, Chamán).

Hábitat: doméstico y peridoméstico.

Triatoma nigromaculata (Stal, 1872) (23) Neiva, $1914\left({ }^{(4)}\right)$

Sinónimos: Eutriatoma nigromaculata Lent \& Pifano, $1939\left(^{6}\right)$; Conorhinus nigromaculatus Stål, $1872 \quad\left({ }^{23}\right)$; Conorhinus variegatus Stål, $1859\left({ }^{9}\right)$.

Distribución geográfica en el mundo $\left({ }^{6}\right)$ : Venezuela (Aragua, Barinas, Bolívar, Cojedes, Lara, Mérida, Monagas, Portuguesa, Sucre, Yaracuy, Delat, Amacuro, Distrito Federal).

Distribución geográfica en el Perú (10,18,19,21): San Martín (Lamas), Cajamarca (Celendín).

Hábitat: Doméstico.
Triatoma infestans infestans $\mathrm{n}$ ssp $\left(^{6}\right)$

Sinónimos: Triatoma infestans erythrophtalmus Noé \& Silva,1949 $\left({ }^{49}\right)$; Conorhinus gracilipes Philippi, $1860\left({ }^{50}\right)$; Conorhinus infestans Berg, $\left.1879{ }^{(30}\right)$; Reduvius infestans Klug en Meyen, $1834\left({ }^{51}\right)$; Triatoma mazzae Jörg, $\left.1937 \quad{ }^{52}\right) ;$ Conorhinus nigrovarius Blanchard, $1890 \quad\left({ }^{53}\right)$; Conorrhinus octotuberculatus Philippi, $1860\left({ }^{50}\right)$; Triatoma sordelli Dios \& Zuccarini, $1926{ }^{\left({ }^{54}\right)}$; Triatoma oswaldoi Neiva \& Pinto, $1923\left({ }^{55}\right)$; Eutriatoma oswaldoi Niño, $1936\left(^{6}\right)$; Conorrhinus paulseni Philippi, 1860 $\left({ }^{50}\right)$; Conorhinus renggeri HerrichSchaeffer, $1848{ }^{(56)}$; Conorhinus sextuberculatus Spinola en Gay, 1852 $\left({ }^{55}\right)$.

Distribución geográfica en el mundo $\left.{ }^{6}\right)$ : Argentina (todas las provincias, excepto Santa Cruz y Tierra del Fuego), Bolivia (Cochabamba, Potosí, Tarija, Beni, La Paz, Chuquisaca, Santa Cruz), Brazil (Alagoas, Bahia, Goiás, Mato Grosso, Mato Grosso del Sur, Minas Gerais, Paraná, Pernambuco, Rio de Janeiro, Rio Grande sel Sur, Santa Catarina, Sao Paulo, Santa Catarina), Chile, México, Paraguay (Concepción, San Pedro, Cordillera, Guairá, Caaguazú, Caazapá, Misiones, Paraguari, Central, Presidente Hayes, Alto Paraguay, Chaco,Nueva Asunción, Boquerón).

Distribución geográfica en el Perú $(2,4,6,14,58)$ : Apurímac (Aymaraes), Ayacucho (Parinacochas, Lucanas), Moquegua (Moquegua, Torata, Samegua, Santa Rosa), Arequipa (Arequipa, Vitor, Castilla, Majes, Camaná), Tacna (Tarata, Locumba), Ica (Ica, Nazca, Palpa), Lima.

Hábitat: doméstico, en algunos lugares peridoméstico. 


\section{DISCUSIÓN}

En la actualidad, se ha comunicado la presencia de triatominos en 23 departamentos del Perú, con la excepción de Huancavelica, en donde solo se ha informado de casos clínicos y no la presencia del vector (Tabla 1). Cajamarca es la que presenta la mayor cantidad de especies. Las especies Belminus peruvianus Herrer, Lent y Wygodzinsky, $1954\left({ }^{7}\right)$; Cavernicola pilosa Barber, $1937\left(^{18}\right)$; Rhodnius ecuadoriensis Lent y León, 1958 $\left({ }^{20}\right)$; Rhodnius pictipes Stål, $1872\left({ }^{23}\right)$; Rhodnius robustus Larrousse, 1927 (26); Eratyrus cuspidatus Stål, $1859\left({ }^{9}\right)$; Eratyrus mucronatus Stål, $1859\left({ }^{9}\right)$; Hermanlentia matsunoi (Fernández Loayza, 1989) Jurberg y Galvão, $1997\left({ }^{28}\right)$; Panstrongylus chinai (Del Ponte, 1929) Pinto, $1931 \quad\left({ }^{17}\right)$; Panstrongylus geniculatus (Latreille, 1811) Pinto, $1931 \quad\left({ }^{17}\right) ; \quad$ Panstrongylus rufotuberculatus (Champion, 1899) $\left({ }^{42}\right)$; Triatoma carrioni Larrouse, $1926\left({ }^{47}\right)$; Triatoma dimidiata (Latreille, 1811) $\left({ }^{35}\right)$ no presentan cambios en la nomenclatura ni en sus descripciones taxonómicas, en base a las revisiones presentadas $\left({ }^{3,5}\right)$.

La presencia de especímenes de Microtriatoma trinidadensis (Lent, 1951) (Prosen y Martínez, 1952) ( ${ }^{15}$ ), en Bolivia y Colombia, fueron consideradas especies separadas, denominándolas también Microtriatoma mansosotoi $\left({ }^{15}\right)$. Luego, se estableció que $M$. mansosotoi es sinonimia de $M$. trinidadensis $\left({ }^{5}\right)$. La presencia de caracteres como color general, tamaño y forma de la cabeza, varían en las poblaciones según el medio donde la especie se desarrolla, lo cual sugiere que se debería llevar a cabo estudios moleculares, para una buena identificación de la especie.

Se considera que Panstrongylus herreri Wygodinsky, $1949\left({ }^{41}\right)$ y Panstrongylus lignarius (Walker, 1873) $\left({ }^{24}\right)$ representan la misma especie. En estudios realizados en morfometría, estas dos especies se encuentran muy relacionadas $\left({ }^{40}\right)$, lo que corrobora los resultados encontrados en estudios citogenéticos y moleculares (secuencia ITS-2 del ADNr nuclear), en los que proponen sinonimia entre estas especies $\left({ }^{59,60}\right)$. Ambas presentan tibias negras, pero $P$. lignarius además presenta una mácula pequeña amarilla sub-basal ( $\left.{ }^{3}\right)$. Probablemente, esta característica diferente se debería al hábitat y orígenes geográficos donde el material fue colectado; proponiéndose dos subespecies Panstrongylus lignarius lignarius y Panstrongylus lignarius herreri $\left.{ }^{(59}\right)$.

Varios autores sugieren que Rhodnius robustus Larrousse, $1927\left({ }^{26}\right)$ y Rhodnius prolixus Stål, $1859\left({ }^{9}\right)$ podrían ser la misma especie con diferentes caracteres, según el lugar de colección; algunos estudios moleculares han demostrado que estas especies están extremadamente relacionadas. En estudios a nivel del ADN mitocondrial con los fragmentos de los genes mtCytB y mtlsurRNA, se ha observado diferencia de $6,8 \%$ y $3,9 \%$, respectivamente $\left({ }^{61}\right)$. Actualmente, se está considerando que algunas poblaciones de $R$. robustus son criptoespecies, no pudiéndose diferenciar por morfología externa. Esta especie, en Venezuela, está cercanamente relacionada a otras poblaciones de la Amazonía $\left({ }^{62}\right)$; lo que sugiere que probablemente $R$. robustus representa un complejo de especies.

Se ha sugerido la nueva subespecie Triatoma infestans infestans $n$ spp $\left({ }^{6}\right)$ debido a la confusión presentada en diferentes poblaciones de $T$. infestans Klug, $1834\left({ }^{51}\right)$, específicamente la población de Misiones (Argentina), que ha sido denominada Triatoma infestans melanosoma Martínez, Olmedo y Carcavallo, $1987\left({ }^{63}\right)$. Se ha considerado $T$. infestans infestans a todas las especies descritas como $T$. infestans Klug, 1834, la cual difiere con la $T$. infestans melanosoma en que presentan patas oscuras, trocánter, base del fémur y base de la tibias amarillas, mancha del conexivo incluyen la 
Contribución al estudio de los triatominos del Perú

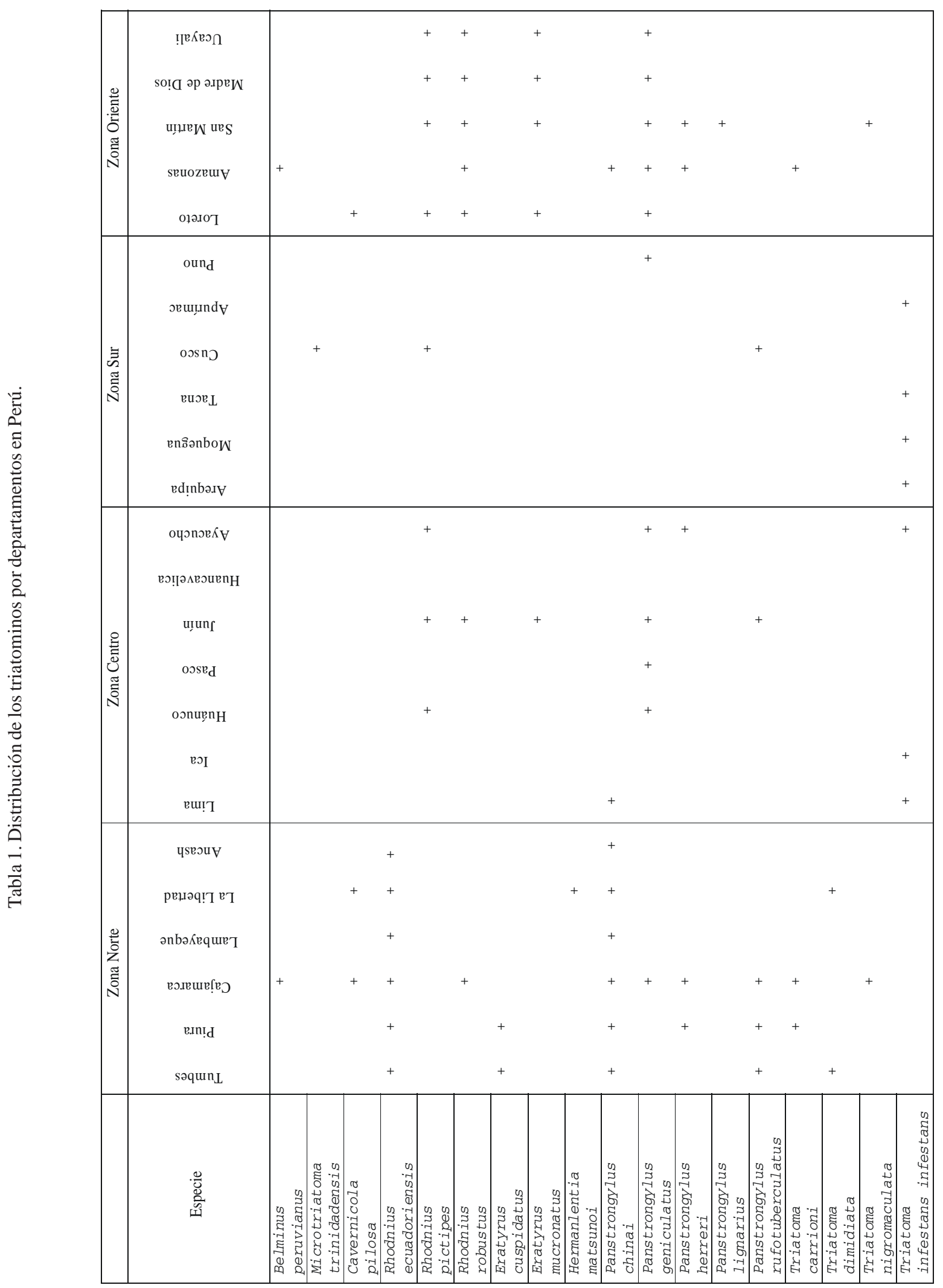

An Fac Med Lima 2006; 67(1) 
sutura y la segunda presenta la base del fémur y base de la tibia de color negro $\left({ }^{3,63}\right)$.

Triatoma nigromaculata (Stål, 1859) $\left({ }^{9}\right)$ ha sido comunicada una sola vez en el Perú ${ }^{(64)}$. La probable afiliación de $T$. nigromaculata al complejo dispar (incluye $T$. dispar, $T$. venosa y $T$. carrioni) puede ayudar a explicar esta comunicación. Aunque las especies presentan un patrón cromático bastante característico, es concebible que una variedad pálida de $T$. carrioni podría haber sido mal clasificada como $T$. nigromaculata. La comunicación merece, sin embargo, investigación adicional $\left({ }^{2}\right)$.

Triatoma venosa (Stål, 1872) Neiva, $1914\left({ }^{44}\right)$ es una especie en duda sobre su presencia en el Perú, ya que está incluida en el complejo dispar. Por lo tanto, se debería realizar estudios moleculares en la especie $T$. carrioni del norte peruano, para aclarar su presencia y distribución en el Perú.

Debido a que todavía existen ciertas interrogantes taxonómicas presentes en algunas especies de la Subfamilia Triatominae, es necesario realizar estudios sobre ecología, morfología, biología molecular y epidemiología molecular, con la finalidad de llegar a una determinación taxonómica precisa de las especies de triatominos del Perú.

\section{REFERENCIAS BIBLIOGRÁFÍCAS}

1. Miles MA. New world Trypanosomiasis. In: Collier L, Balows A, Sussman M (eds.). Microbiology and Microbial Infections. Vol 5: Parasitology. UK: Topley y Wilson's; 1998.

2. Cuba CA, Abad-Franch F, Roldán J, Vargas F, Pollack L, Miles M. The Triatomines of northern Perú, with emphasis on the ecology and infection by Trypanosomes of Rhodnius ecuadoriensis (Triatominae). Memorias del Instituto Oswaldo Cruz. 2002;97(2):175-83.

3. Elliot A, Cáceres I, Guillén Z, Nakashima I. Identificación de los chinches triatominos (Hemiptera, Reduviidae) conocidos del Perú. Revista Peruana de Entomología. 1989;31:18-20.

4. Carrasco F. Contribución al estudio de Triatoma infestans (Reduviidae, Heteroptera) en el Perú. Revista Peruana de Entomología. 1970;13(1):9-14.

5. Lent H, Wygodzinsky P. Revision of the Triatominae (Hemiptera, Reduviidae), and their significance as vectors of Chagas disease. Bulletin of the American Museum of Natural History. 1979; 163: 123-520.

6. Galvão C, Carcavallo R, Da Silva D, Jurberg J. A checklist of the current valid species of the subfamily Triatominae Jeannel, 1919 (Hemiptera, Reduvidae) and their geographical distribution, with nomenclatural and taxonomic studies notes. Zootaxa. 2003;202:1-36.

7. Herrer A, Lent H, Wygodzinsky P. Contribución al conocimiento del género Belminus Stål, 1859 (Triatominae, Reduviidae, Hemíptera). Anales del Instituto de Medicina Regional, Tucumán. 1954;4:85-105.

8. Usinger RL. The Triatominae of North and Central America and the West Indies and their public health significance. US Public Health Bulletin. 1944; 288: 1-83.

9. Stål C. Monographie dar Gattung Conorhinus und Werwandten. Berliner Entomologische Zeitschrift. 1859;3: 99-117.

10. Distant W. Rhynchotal notes: XIV. Heteroptera: families Hydrometidae, Henicocephalidae, and Reduviidae (part). Annual Magazine of Natural History, London. 1904;10(7).

11. Escalante H, López E, Jara C, Torres P. Distribución de la Enfermedad de Chagas en la zona norte del Perú. REBIOL. $1997 ; 17(1$ y 2):115-27.

12. Guillén Z, Cáceres I, Elliot A, Ramírez J. Triatominos del norte peruano y su importancia como vectores de Trypanosoma spp. Revista Peruana de Entomología. 1988;31:25-30.

13. Guillén Z, Cáceres I, Elliot A, Ramírez J. Distribución geográfica de los triatominos en el oriente del Perú. Revista Peruana de Medicina Tropical, UNMSM. 1992;6:93-7.

14. Lumbreras H. El problema de la enfermedad de Chagas en los diferentes departamentos del Perú. Revista Viernes Médico. 1972; Vol XXIII Nº1: 43-77.

15. Prosen AF, Martínez A. Un nuevo género y especie de Triatominae (Hemíptera, Reduviidae). MEPRA Publicación. 1952;23:5-12.

16. Lent H. Segunda especie del género Bolbodera Valdés, 1910 (Hemíptera, Reduviidae). Revista Brasileira de Biologia. 1951;11:153-6.

17. Pinto C. Valor do rostro e antennas na caracterizacao dos géneros do triatomideos. Hemiptera, Reduvioideo. Boletim Biológico de São Paulo. 1931;19:45-136.

18. Barber HG. A new bat-cave bug from Panamá (Hemiptera: Reduviidae). Proceedings of the Entomological Society of Washington. 1937;39:60-3.

19. Pinto C. Triatomideos de Venezuela, com a descripção de uma nova espécie do gênero Eutriatoma. Annais da Faculdade de Medicina de São Paulo. 1926;1:85-7. 
20. Lent H, León L. Um novo Rhodnius Stäl do Ecuador (Hemiptera, Reduvidae). Revista Brasileira de Biologia. 1958; 18:181-5.

21. Cáceres A, Troyes L, Gonzáles-Pérez A, Llontop E, Bonilla C, Murias E, et al. Enfermedad de Chagas en la región nororiental del Perú. I. Triatominos (Hemiptera: Reduvidae) presentes en Cajamarca y Amazonas. Revista Peruana de Medicina Experimental y Salud Pública. 2002;19(1):17-23.

22. Varas J, Vargas F, Roldán J, López O, Obeso S, Ampuero C. Determinación de la variación morfométrica entre poblaciones de Rhodnius ecuadoriensis procedentes de habitats naturales y de insectario. V Congreso Peruano de Parasitología. 2002. 32pp.

23. Stål C. Enumeratio Hemipterorum. Pars 2. Kongliga Svenska Vetenskaps-Akademiens Handlingar. 1872;10:1-159.

24. Walker F. Catalogue of the specimens of Hemiptera Heteroptera in the collection of the British Museum. London: $\mathrm{BM}(\mathrm{NH}) ; 1873$. Part 7 y Part 8.

25. Guillén Z, Cáceres I, Elliot A. Los triatominae (Hemiptera, Reduvidae) de la zona centro del Perú. Revista Peruana de Medicina Tropical, UNMSM. 1992;6:89-91.

26. Larrousse F. Etude biologique et systématique du genre Rhodnius Stål (Hemiptères, Reduviidae). Annales de Parasitologie. 1927;5:63-88.

27. Jeannel R. Insectes Hémiptères III. Hemicocephalidae et Reduviidae. En: Voyage de Ch. Allaucaud et R. Jeannelen Afrique Orientale (1911-1912). Paris: Résultats Scientifiques; 1919.

28. Jurberg J, Galvão C. Hermanlentia n. gen du tribo Triatomini, com um rol de espécies de Triatominae (Hemiptera, Reduvidae). Memorias de Instituto Oswaldo Cruz. 1997;92:181-5.

29. Fernández R. Triatoma matsunoi nueva especie del norte peruano (Hemiptera, Reduvidae, Triatominae). Revista Peruana de Entomología. 1989;31:21-4.

30. Berg C. Hemiptera Argentina enumeravit especiesque novas. Buenos Aires: P.E.Coni; 1879.

31. Kirkaldy G. Bibliographical and nomenclatural notes on the Hemiptera. $N^{0}$ 3. The Entomologist. 1904;37:279-83.

32. Del Ponte E. Algunas especies nuevas del género Triatoma Lap. Boletín de la Sociedad Entomológica Argentina. 1929;1:3-8.

33. Valderrama A, Lizano E, Cabello D, Valera M. Panstrongylus turpiali n.sp. (Hemiptera: Reduviidae: Triatominae) from Venezuela. Caribbean Journal of science. 1996;32:142-4

34. Juárez S, Silva T, Celerón W. Panstrongylus chinai intradomiciliario en Nieto y La Peña distritos de Salas, Lambayeque, Perú. Abril-Diciembre 2001. V Congreso Peruano de Parasitología. 2002; 31pp.

35. Latreille PA. Insectes de lí Amérique recuetilis pendant le voyage de MM. de Humboldt et Bonpland. In: Humboldt A and Bonpland A. Voyage aux régions équinoxiales du nouveau continent, París Vol 1; 1811.
36. Erichson W. Insecten. In: Schomburgk R. Resen in BritischGuiana in der jahren 1840-1844 im aufrag Sr Majestat des Konings von Pruessen. Weber, Leipzig; 1848.

37. Neiva A, Pinto C. De um novo hemiptero hematophago brasileiro (Triatoma fluminensis nov. sp). Brasil-Médico. 1922;36:402-3.

38. Chagas C. Sobre um Trypanosoma do tatú, Tatusia novemcincta, transmitido pela Triatoma geniculata Latr. (1811). Possibilidade de ser o tatú um depositario do Trypanosoma cruzi no mundo exterior (Nota previa). BrasilMédico. 1912;26:305-6.

39. Ortiz I. Sobre algunos arreglos taxonómicos en el orden de los Hemiptera (Insecta) con referencia especial a la identificación de la familia Triatomidae Pinto, 1931. Revisión del género Panstrongylus Berg, 1875 y descripción de una nueva especie (Panstrongylus parageniculatus $\mathrm{n}$. sp.). Revista del Instituto de Higiene, Caracas. 1971;4:49-87.

40. Magalhães C, Jurberg J, Galvão C, Da Silva D, Rodríguez J. Estudo morfométrico do Gênero Panstrongylus Berg, 1879 (Hemiptera, Reduviidae, Triatominae). Memoria del Instituto Oswaldo Cruz. 2003;98(7):939-44.

41. Wygodzinsky P. Sobre Panstrongylus del Perú, con la descripción de una nueva especie (Triatominae, Reduvidae, Hemiptera). An Inst Med Reg Tucumán. 1948:2;197-208.

42. Champion G. Insecta Rhynchota. Hemiptera-Heteroptera. Biología Centrali-Americana. 1899;2(14): 229-43.

43. Campos F. Una nueva especie del género Triatoma (Hemiptero Heteroptero de la fam. Reduviidae, subfam. Acanthaspidinae). Revista del Colegio Nacional Vicente Rocafuerte, Guayaquil. 1932;14:3-6.

44. Neiva A. Revisão do gênero Triatoma Lap. Thesis, Faculdade de Medicina, Rio de Janeiro. 1914. 80pp.

45. Usinger RL. Descriptions of new Triatominae with a key to genera (Hemiptera, Reduviidae). University of California Publications in Entomology. 1939;7:33-56.

46. Laporte F. Essai diune classification systematique de liordre des Hémiptères (Hémiptères, Hétéroptères, Latr.). Magazine de Zoologie. 1832/3;1:1-88.

47. Larrouse F. Description de deux espéces nouvelles du genre Triatoma: $T$. carrioni $\mathrm{n}$. sp. et $T$. pintoi n.sp. Annales de Parasitologie Humaine et Comparé. 1926;4:136-9.

48. Usinger RL. Notes and descriptions of neotropical Triatominae (Hemiptera, Reduviidae). Pan-Pacific Entomologist. 1941;17:49-57.

49. Noé J, Silva R. Una nueva variedad de Triatoma infestans Klug, 1834. Boletín de Informaciones Parasitológicas Chilenas. 1949;4:4-5.

50. Philippi RA. Viage al Desierto de Atacama. Chile: Libreria Anton, Sajonia; 1860.

51. Klug J. In Meyen FJF. Reise um die Erde, in der Jahren 1830, 1831 und 1832, ausgefürht von FJF Meyen. CW Eichhoff, Berlín; 1834.

52. Jörg M. Triatoma mazzae nova species de Triatomidae (Hemiptera-Heteroptera, reduvioidea) de Argentina. MEPRA Publicación. 1937;37:33-47. 
53. Blanchard R. Traité de zoologie médicale. París: Balliere et Fils.; 1890. p. 2.

54. Dios R, Zuccarini J. Sobre la existencia del Triatoma oswaldoi Neiva y Pinto en la República Argentina. Revista de la Sociedad Argentina de Biología. 1926;2:179-80.

55. Neiva A, Pinto C. Dos reduvideos hematophagos encontrados do Distrito Federal E Estado do Rio de Janeiro, com a descripção de uma espécie nova. Brasil-Médico. 1923;37:457.

56. Herrich-Schaeffer G. Die Wanzenartigen. Insekten. C.H. Zehschen Buchhandlung, Nurenberg. 1848;8.

57. Spinola M. Hemípteros. En: Gay. Historia Física y Política de Chile. 1852;7:113-320.

58. Ruelas N, Vásquez L, Ayaqui R, Martínez E, Mollepaza M. Algunos aspectos epidemiológicos de la enfermedad de Chagas e indicadores entomológicos en 4 localidades del Valle de Majes-Arequipa. V Congreso Peruano de Parasitología. 2002. 24pp.

59. Marcilla A., Bargues MD, Abad-Franch F, Panzera F, Carcavallo RU, Noireau F, Galvão C, Miles M, Dujardin JP, Mas-Coma S. Nuclear rDNA ITS-2 sequences reveal polyphyly of Panstrongylus species (Hemiptera: Reduviidae: Triatominae), vectors of Trypanosoma cruzi. Infection, Genetics and Evolution. 2002;1:225-335.

60. Pérez R, Hernández M, Noel M, Rose V, Valente S, Da Costa V, Moreno J, Angulo V, Sandoval C, Roldán J, Vargas F, Wolff M, Panzera F. Cromosomal evolution trends of the genus Panstrongylus (Hemiptera, Reduviidae), vector Chagas disease. Infection, Genetics and Evolution. 2002;2:47-56.
61. Lyman D, Monteiro F, Escalante A, Cordón-Rosales C, Wesson D, Dujardin JP, et al. Mithocondrial DNA sequence Variation among Triatomine vectors of Chaga's disease. American Journal of Tropical Medicine and Hygiene. 1999;60(3):377-86.

62. Monteiro FA, Barrett TV, Fitzpatrick F, Cordón-Rosales C, Feliciangeli D, Beard CB. Molecular phylogeography of the Amazonian Chagas disease vectors Rhodnius prolixus $y$ R. robustus. Molecular Ecology. 2003;12:997-1006.

63. Martínez A, Olmedo RA, Carcavallo RU. Una nueva subespecie Argentina de Triatoma infestans. Chagas. 1987;4:7-8.

64. Calderón G, Monzón L. Primer hallazgo de Triatoma nigromaculata (Stål, 1872) en el Perú. Revista Peruana de Entomología. 1995;37:124.

Manuscrito recibido el 06 de noviembre de 2005 y aceptado para publicación el 06 febrero de 2006.

Correspondencia: Blgo. Julio César Chávez Galarza Instituto de Investigación en Microbiología y Parasitología Tropical Universidad Nacional de Trujillo

Av. EE UU 288 Urb. El Recreo

Trujillo, Perú.

Correo-e: alucard_jul@hotmail.com 